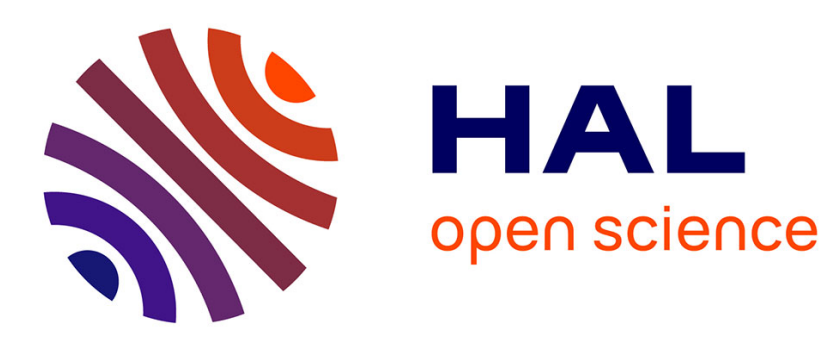

\title{
Evolving Specific Network Statistical Properties using a Gene Regulatory Network Model
}

\author{
Miguel Nicolau, Marc Schoenauer
}

\section{To cite this version:}

Miguel Nicolau, Marc Schoenauer. Evolving Specific Network Statistical Properties using a Gene Regulatory Network Model. Genetic and Evolutionary Computation Conference (GECCO), Jul 2009, Montreal, Canada. inria-00377089

\section{HAL Id: inria-00377089 \\ https://hal.inria.fr/inria-00377089}

Submitted on 21 Apr 2009

HAL is a multi-disciplinary open access archive for the deposit and dissemination of scientific research documents, whether they are published or not. The documents may come from teaching and research institutions in France or abroad, or from public or private research centers.
L'archive ouverte pluridisciplinaire HAL, est destinée au dépôt et à la diffusion de documents scientifiques de niveau recherche, publiés ou non, émanant des établissements d'enseignement et de recherche français ou étrangers, des laboratoires publics ou privés. 


\section{Evolving Specific Network Statistical Properties using a Gene Regulatory Network Model}

\author{
Miguel Nicolau \\ INRIA Saclay - Île-de-France \\ LRI - Batîment 490 \\ Université Paris-SUD \\ 91405 Orsay Cedex, France \\ Miguel.Nicolau@inria.fr
}

\author{
Marc Schoenauer \\ INRIA Saclay - Île-de-France \\ LRI - Batîment 490 \\ Université Paris-SUD \\ 91405 Orsay Cedex, France \\ Marc.Schoenauer@inria.fr
}

\begin{abstract}
The generation of network topologies with specific, userspecified statistical properties is addressed using an Evolutionary Algorithm that is seeded by an Artificial Gene Regulatory Network Model. The work presented here extends previous work where the proposed approach was demonstrated to be able to evolve scale-free topologies. The present results reinforce the applicability of the proposed method, showing that the evolution of small-world topologies is also possible, but requires a carefully crafted fitness function.
\end{abstract}

\section{Categories and Subject Descriptors}

C.2.1 [Computer Systems Organization]: Network Architecture and Design: Network topology

; I.2.8 [Computing Methodologies]: Artificial Intelligence: Problem Solving, Control Methods, and Search

\section{General Terms}

Algorithms

\section{Keywords}

Regulatory Networks, Small-world Topology, Evolutionary Computation

\section{INTRODUCTION}

Network generative procedures targeted toward specific topology properties are generally either iterative processes that sequentially add nodes using problem-dependent rules $[22,3]$, or ad hoc stochastic procedures modifying existing networks [16]. However, such procedures need to be designed anew whenever a new target property is wanted. Goal-directed procedures, on the other hand, only require a measure of the desired properties, and some (generally stochastic) optimisation method able to search the space of

Permission to make digital or hard copies of all or part of this work for personal or classroom use is granted without fee provided that copies are not made or distributed for profit or commercial advantage and that copies bear this notice and the full citation on the first page. To copy otherwise, to republish, to post on servers or to redistribute to lists, requires prior specific permission and/or a fee.

GECCO'08, July 12-16, 2008, Atlanta, Georgia, USA.

Copyright 2008 ACM 978-1-60558-131-6/08/07 ...\$5.00. network topologies. Unfortunately, such approaches generally suffer from two main drawbacks: the curse of dimensionality, making it intractable to optimize large networks, and the bootstrap problem, in that random topologies barely indicate any meaningful path toward good solutions of the problem at hand.

An alternative is to use specific initialisation techniques, producing diverse (but not random) topologies, which can then be used as a starting point for the evolutionary process. Artificial Genetic Regulatory Networks (GRNs) offer such a procedure: previous work [15] has demonstrated that simple models of GRNs were able to boost the evolutionary optimisation of topologies in order to create scale-free topologies.

Building on this work, the present paper uses similar ideas to extend the generality of the approach, and introduces an original generative procedure to design Small World topologies [21]. This is a specific kind of topology, where most nodes are not directly connected, yet the average connection distance between any two nodes is very low. This kind of topology has been shown to exist in many real-life networks, such as biological transcriptional networks [20], computer networks [4], or social networks [8].

The generation of specific network topologies allows their incorporation in a variety of systems. Although regular and random topologies have been shown to work well in several fields, such as parallel and distributed computing [14] or simple automata [5], other systems have been shown to profit from specific topologies, such as large cellular automata systems [21], and evolutionary algorithms of different classes [7, $6,17,18]$. The objective of the current work is thus to generate topologies that are to be tested in other optimisation algorithms, such as Echo-State networks [10, 12].

The results obtained in the current study show that the presented method is applicable to the evolution of smallworld networks, therefore reinforcing the usability of such approaches to the design of network topologies with userspecified statistical properties.

This paper is structured as follows: Section 2 presents the gene regulatory model used, including its representation, and initialisation technique; Section 3 introduces the definition of small-world topologies, and the method used to extract topologies from the regulatory model. Then Section 4 defines the objective to be achieved, and presents and analyses the results obtained, showing the advantage of using the presented initialisation technique. Finally, section 5 draws conclusions and future work directions. 


\section{THE GENE REGULATORY NETWORK MODEL}

The expression of genes in a genome is regulated by Transcription Factors. These are special proteins, produced by other genes, which can enhance or inhibit the production of their target genes; the networks of interactions between genes and the proteins they produce are termed Gene Regulatory Networks (GRN).

The model used in this work was first proposed by Wolfgang Banzhaf [2]. It represents a genome as a bit string, and uses specific bit sequences as promoter sites, identifying the location of a gene. If such a site is found (thus identifying a gene), the $5 \times 32$ bits following it represent the protein that this gene produces, and the the $2 \times 32$ bits upstream from the promoter site represent its enhancer and inhibitor sites, respectively. Fig. 1 illustrates the model.

The promoter site can be any sequence of bits; in this case, it is the sequence XYZ01010101, where $X, Y$ and $Z$ are any 8 bit sequence. The protein produced by the gene is a 32 bit binary sequence, extracted by a majority rule between all 5 sequences of 32 bits that compose it (that is, if 3 or more equally located bits are set to 1 , then the corresponding bit in the protein is set to 1 ).

In this model, all proteins produced are transcription factors, and therefore they all regulate the expression of all genes, including their own producing gene. Regulation works by matching a protein and the regulating sites of a gene with the XOR operation: the result is the regulating strength. The enhancing and inhibiting signals regulating the production of protein $p_{i}$ are then calculated as:

$$
e_{i}, h_{i}=\frac{1}{N} \sum_{j=1}^{N} c_{j} \exp \left(\beta\left(u_{i, j}-u_{i, \max }\right)\right)
$$

where $N$ is the number of proteins, $c_{j}$ is the concentration of protein $j, u_{i, j}$ is the XOR result between the regulating site of gene $i$ and protein $j, u_{i, \max }$ is the maximum match achieved for gene $i$, and $\beta$ is a positive scaling factor.

Given these signals, the production of protein $i$ is calculated via the following differential equation:

$$
\frac{d c_{i}}{d t}=\delta\left(e_{i}-h_{i}\right) c_{i}-\Phi
$$

where $\delta$ is a positive scaling factor (representing a time unit), and $\Phi$ is a term that proportionally scales protein production, ensuring that $\sum_{i} c_{i}=1$ at all times, which results in competition between binding sites for proteins.

\subsection{Initialisation Method}

Although the binary genomes used within the model can be randomly created, an initialisation method has been proposed [2], based on a Duplication and Mutation (DM) process. It involves creating a random 32 bit sequence, followed by a series of length duplications associated with a low mutation rate. It has been shown [23] that this process of growing genomes can also occur in nature.

\section{NETWORK TOPOLOGIES}

Even though the model used is overly simplified compared to what is known of biological GRNs, an interesting issue is to find out whether or not the resulting interaction network exhibits particular properties resembling those found in natural networks. Previous work [15] has shown that evolution of linear genomes to achieve Scale-Free topologies [24, 11, 9, $20,1]$ is possible; the current work concentrates on SmallWorld topologies $[21,20]$.

\subsection{Small-World Networks}

A small-World network is a network topology where most nodes are not directly connected to each other, yet the average distance between any two nodes is very low. Albeit closely related to scale-free topologies, small-world topologies are based on completely different statistical measurements.

Watts [21] describes them in terms of their characteristic path length $L$, and clustering coefficient $C$, with $C_{i}$ being the percentage of all nodes connected to node $i$ that are also connected to each other ${ }^{1}$. Given these definitions, a graph with $n$ nodes and average vertex degree $k$ is a small-world network, if it satisfies the following conditions:

$$
L \geq L_{\text {rand }} \sim \frac{\ln (n)}{\ln (k)} \quad C \gg C_{\text {rand }} \sim \frac{k}{n}
$$

where $n \gg k \gg \ln (n) \gg 1$.

This topology has been shown to exist in many real-life networks, such as biological transcriptional networks [20], computer networks [4], and social networks [8].

The typical generative model to build small-world topologies consists in the rewiring of the connections of a regular network, to introduce increasing randomness [22]. Part of the motivation of the current work lies in providing an alternate means of generating such topologies, which does not rely on direct manipulations of the network connectivity.

\subsection{Artificial Regulatory Networks}

Once a genome has been constructed with the model described (see Section 2), its regulatory network topology can be analysed: genes will be represented as nodes, and the proteins they produce will be directed edges towards the genes they regulate; the weight of those edges will be the number of complementary bits between the regulating protein and the target gene (as seen in Eq. 1).

As all produced proteins regulate all genes, the resulting graph is complete. However, a threshold can be set on the minimum weight a connection must have, before being considered as an edge on the resulting regulating network.

Using different regulation thresholds will therefore result in different networks. For example, Fig. 2 and 3 show regulating networks of the same random genome for two different threshold values (23 and 24 , out of 32 ). While almost all nodes are connected in Fig. 2, increasing the threshold by one removes many connections, and the graph in Fig. 3 is only a small sub-graph of the previous one (nodes which become isolated are not shown, which explains the smaller number of genes).

Networks extracted from genomes initialised with the DM technique have remarkably different topologies, as seen in Fig. 4. The use of DM steps with low mutation result in a much shallower hierarchy of genes, with a few master genes, and most other genes poorly connected. Increasing the threshold removes connections, but the same master genes are still present, as can be seen in Fig. 5 .

\footnotetext{
${ }^{1}$ The phenomenon $C_{i}=1.0$, where "all my friends are each other's friends", is also known as a clique.
} 


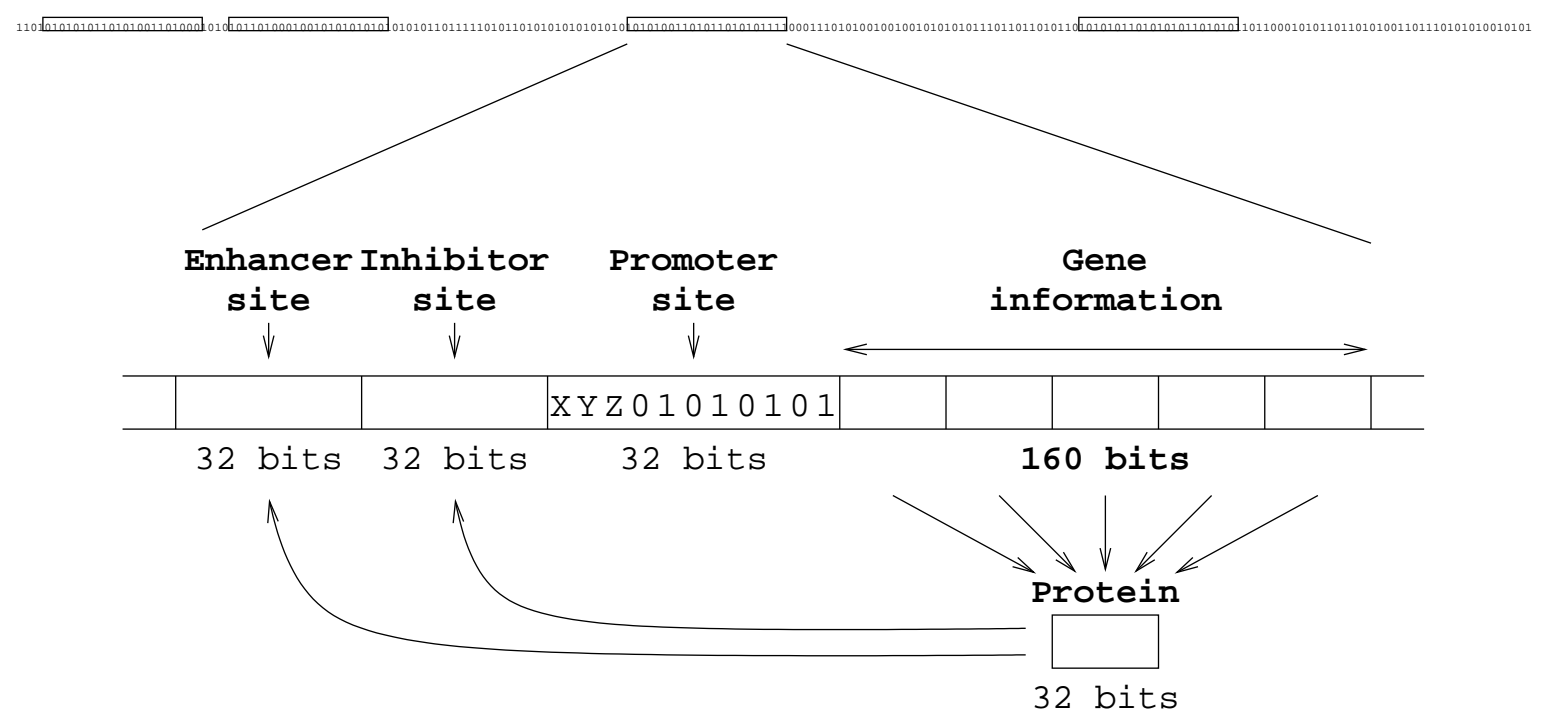

Figure 1: Close-up view of the representation of a gene within the model.

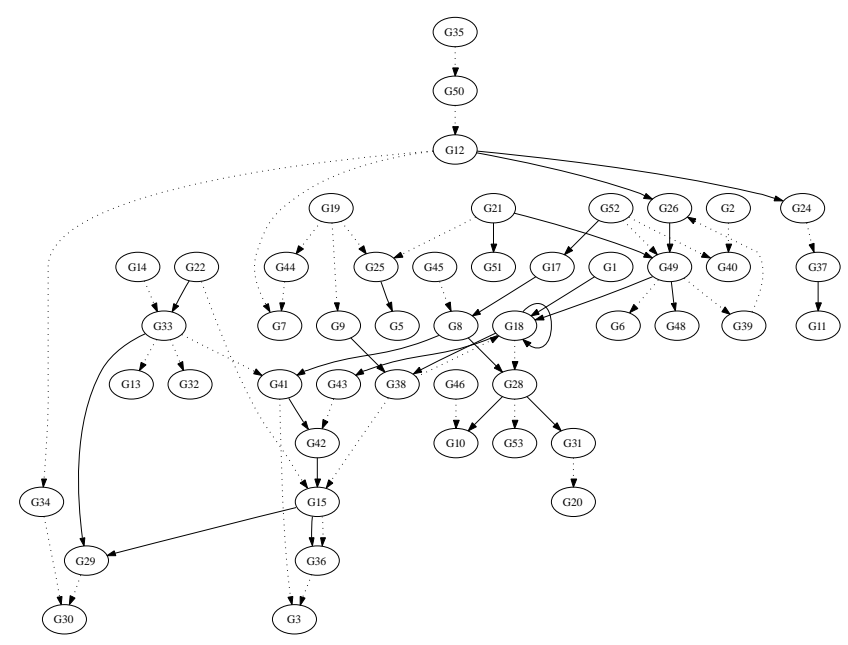

Figure 2: Regulatory network for a random genome of length $L_{G}=32,768$, at a threshold of 23 bits. Solid edges are enhancing interactions, dotted edges are inhibiting ones.

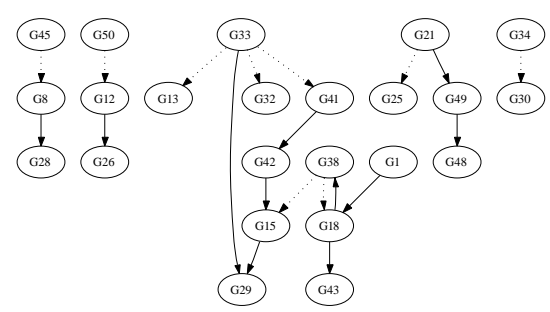

Figure 3: Gene regulatory network for the genome from Fig. 2, at a threshold of 24 bits.

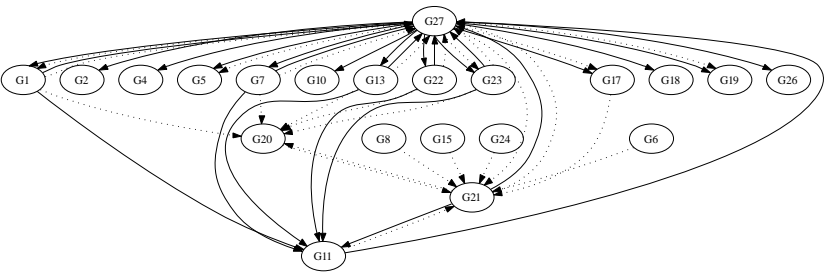

Figure 4: Gene regulatory network for a genome of length $L_{G}=32,768$, created using 10 duplication events and a mutation rate of $1 \%$, at a threshold of 16 bits.

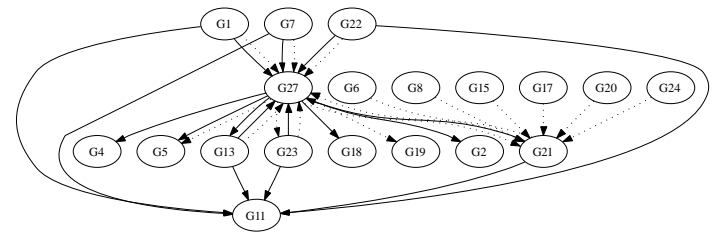

Figure 5: Gene regulatory network for the genome from Fig. 4, at a threshold of 17 bits.

\subsection{Connectivity variance}

In order to analyse the effect of the threshold value on the network connectivity, an approach similar to that of Kuo et al. [13] has been used: 100 genomes have been generated, using 14 duplication events, and the network connectivity (fraction of edges) has been computed for each threshold.

The network connectivity is defined as:

$$
N C=\frac{\# \text { edges }}{2 n^{2}}
$$

where \#edges is the number of edges in the network, and $n$ is the number of nodes $\left(2 n^{2}\right.$ thus being the maximum number of possible edges). 
Fig. 6 shows the connectivity as a function of the threshold, for mutation rates of $1 \%, 5 \%, 10 \%$, and $50 \%$. It is a clear illustration of the very different behaviors with respect to connectivity depending on the mutation rate used during the DM process:

- A high mutation rate (or, equivalently, the completely random generation of the genome) creates a network which stays fully connected with a wide range of threshold values; then, there is a sharp transition to no connectivity. Moreover, there is a very small variance between different networks.

- A low mutation rate creates a network which quickly loses full connectivity; however, its transition to no connectivity is much smoother than that of random networks. Moreover, there is very large variance between different networks generated with the same mutation rate.

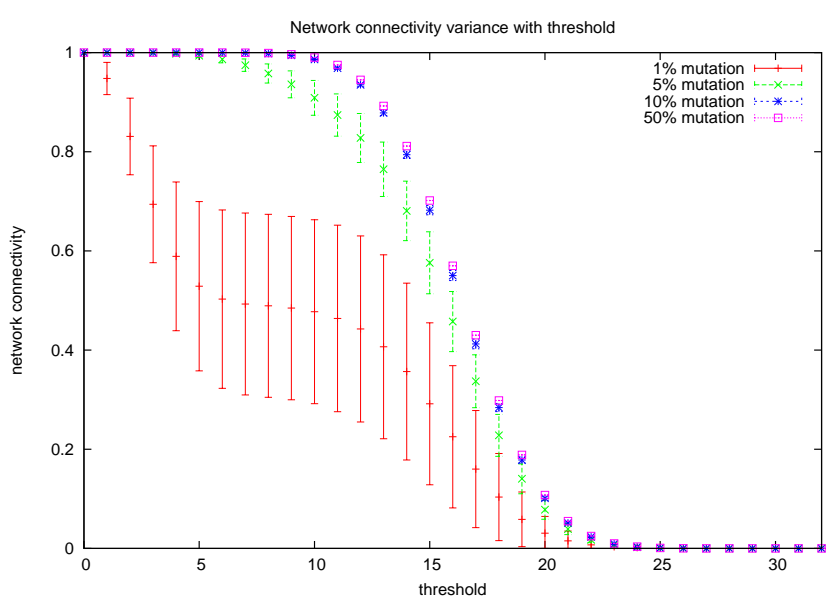

Figure 6: Fraction of edges in a graph as compared to a fully connected network (and standard deviations), versus threshold parameter, based on samples of 100 genomes, created using 14 duplication events, and mutation rates of $1 \%, 5 \%, 10 \%$, and $50 \%$.

\section{EVOLUTION OF STATISTICAL PROP- ERTIES}

The objective of this section is to test whether genomes created with the model can be evolved, such that their regulating networks exhibit precise statistical properties, consistent with the definition of small-world topologies.

\subsection{The Evolutionary Algorithm}

A population of bit-string genomes is evolved, using the simple bit-flip mutation as the only variation operator. The evolution is a straightforward $(25+25)-E S$ : 25 parents give birth to 25 offspring, and the best 25 of the 50 parents+offspring become the parents of next generation. The mutation rate is adapted following the 1/5 rule of Evolution Strategies [19]: its rate is initially set to $1 \%$ (per bit), and when the rate of successful mutations is higher than $1 / 5$ (i.e. when more than $20 \%$ mutation events result in an increase of fitness), the mutation rate is doubled; it is halved in the opposite case ${ }^{2}$.

In order to compare the evolvability of DM initialised genomes and completely random populations, 50 independent runs of 50 generations were performed with each type of genome, for each of the following fitness functions.

\subsection{Fitness Function}

In order to satisfy the relationship $n \gg k \gg \ln (n) \gg 1$ (seen in Section 3.1), candidate objective functions should try to minimise the following three (normalized) terms:

$$
\frac{n}{n-k} ; \frac{k}{k-\ln (n)} ; \frac{\ln (n)}{\ln (n)-1}
$$

Furthermore, in order to be considered small-world, a network must satisfy $L \geq L_{\text {rand }}$, and $C \gg C_{\text {rand }}$, so networks with $C \leq C_{\text {rand }}$ should be discarded, and the following terms should be minimised:

$$
\left(L-L_{\text {rand }}\right)^{+} ; \frac{C}{C-C_{\text {rand }}}
$$

A possible fitness function for an Evolutionary Algorithm is thus given by a weighted sum of those terms, whose weights should be tailored to the problem at hand:

$$
\begin{aligned}
F(x) & =\alpha_{1} \frac{n}{n-k}+\alpha_{2} \frac{k}{k-\ln (n)}+\alpha_{3} \frac{\ln (n)}{\ln (n)-1}+ \\
& +\alpha_{4}\left(L-L_{\text {rand }}\right)^{+}+\alpha_{5} \frac{C}{C-C_{\text {rand }}}
\end{aligned}
$$

Note that, in the current work, all weights were set to 1 .

\subsection{Results}

Fig. 7 shows the best fitness in the population averaged over the 50 runs, for both initialisations procedures, using both fitness cases.

The results obtained show that genomes initialised with the DM technique get off their starting blocks with topologies which are much closer to being small-world, as defined in equation 4. Not only that, but their structure actually allows them to continuously improve their fitness score through evolution.

Random genomes, on the other hand, have starting topologies which score badly. Furthermore, their regular topologies have very similar scores across different runs, and show little signs of evolution over time.

Table 1 shows the relevant statistical measures of random and DM initialised networks, both without or with evolution. Of relevant interest is the fact that, after evolution, DM initialised networks exhibit a high clustering coefficient, while keeping a characteristic path length similar to that of randomly initialised genomes.

\subsection{Analysis}

The shallow hierarchies observed in initialised genomes exhibit characteristics similar to those of Small-World topologies, leading to the results observed. To analyse the reasons

\footnotetext{
${ }^{2}$ Because of the possibility of neutral mutations (especially with low mutation rates), if there were more than $50 \%$ neutral mutations, the rate was doubled in any case.
} 
Table 1: Initial and evolved results, for random and DM initialised genomes.

\begin{tabular}{|c|c|c|c|c|c|c|c|c|}
\hline & Generation & Threshold & $\mathrm{N}$ & $\mathrm{k}$ & $\ln (\mathrm{N})$ & $\mathrm{L}$ & $\mathrm{C}$ & Fitness \\
\hline Random & 0 & 22.199 & 1072.400 & 129.300 & 6.978 & 2.016 & 0.121 & $\mathbf{2 . 8 1 2}$ \\
\cline { 2 - 9 } Genomes & 50 & 23.000 & 1093.099 & 42.899 & 6.996 & 2.140 & 0.040 & $\mathbf{2 . 6 6 2}$ \\
\hline Initialised & 0 & 12.900 & 1910.799 & 90.700 & 7.554 & 1.962 & 0.404 & $\mathbf{2 . 2 7 4}$ \\
\cline { 2 - 10 } Genomes & 50 & 11.699 & 1782.400 & 44.399 & 7.479 & 1.976 & 0.810 & $\mathbf{1 . 9 3 6}$ \\
\hline
\end{tabular}

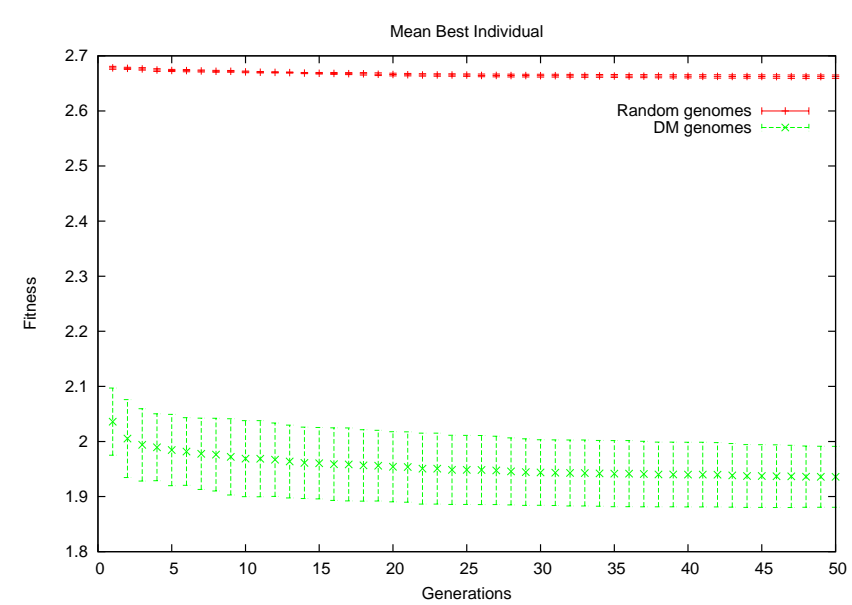

Figure 7: Average best fitness per generation across 50 independent runs, for random and 1\% DM initialised genomes. Error bars plot the standard deviation across runs.

leading to such a difference in the extracted network topologies, a sequence of DM steps was analysed, as it took place.

The original random 32 bit sequence was as follows:

\section{1}

This sequence was then subjected to a series of DM events, with a probability of mutation of $1 \%$ per bit. After 6 DM events, the first gene appeared, and after 7 events, there are already four genes. The resulting networks were extracted (Fig. 8 and 9) using 13 as the connection threshold ${ }^{3}$.

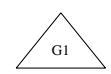

Figure 8: Gene network after 6 duplication events.

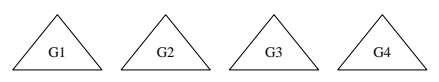

Figure 9: Gene network after 7 duplication events.

The shape of the genes determines their starting location, when mapped to the original 32 bit sequence. For example,

${ }^{3}$ This value was chosen deliberately, based on the resulting network after all DM events, to illustrate the propose discussed. for the genome in Fig. 9, the starting locations for its genes were bits 905, 1929, 2377 and 2761, respectively. If we divide these by 32 and take the remainder, we see that they all start at the $9^{\text {th }}$ bit of a duplication of the original sequence, so they are all represented by the same (triangular) shape.

This also explains why there are no connections between genes in Fig. 9. As all genes originate from the same initial sequence of bits, the few mutations that occurred during the 7 DM steps did not create enough differences between regulating sites and produced proteins, to trigger a connection at threshold 13 .

After the $8^{\text {th }}$ DM event, the network takes on a different topology (Fig. 10). Most genes are still duplications of the $9^{t h}$ bit of the original sequence; however, G7 starts at a different location, and is thus represented by a different (rectangular) shape.

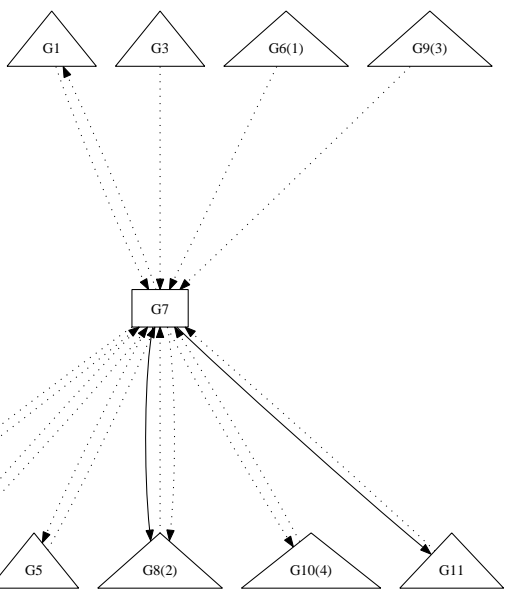

Figure 10: Gene network after 8 duplication events.

As the connectivity between genes is established by the difference between regulation sites and proteins, genes originating from different locations are more likely to be connected, even using lower threshold values. This can be seen in Fig. 10: genes labelled with equal shapes do not connect to each other.

In this DM step one can also see pure duplications of genes, that is, genes that are created as duplications of other genes appearing upstream in the genome sequence: in those cases, the genes are labelled with their originating gene between brackets (e.g. G6(1)). But even pure duplications can generate slightly different genes, because mutation events 
can occur during the duplication process. G6(1) is an example: it only has an outward inhibiting connection to $\mathbf{G 7}$, whereas $\mathbf{G 1}$ also has an inward inhibiting connection originating from the same gene.

With 9 DM steps, the network becomes a lot more complex (Fig. 11). There are still only two relative gene origins (triangles and rectangles), but either through pure duplications or discovery of new genes, there are now 25 genes.

One can see that triangles still do not connect with rectangles (due to the threshold value chosen). Therefore, since there are a lot more triangles than rectangles, the latter become highly connected, and can be seen acting as connection hubs.

Finally, a last DM step is performed (Fig. 12), creating a network with 50 genes. Although hard to analyse for the naked eye, one can clearly see its shallow hierarchy, with a few highly connected nodes, to which most other nodes connect. One can also see the appearance of a third type of gene, labelled with a pentagon shape, which becomes the most connected gene. Table 2 shows a list of the gene families, along with their count, initial location, corresponding initial bit, and average number of inward, outward, and total connections.

Although this network is just an example, many networks were found to follow the same mechanics while being extracted from genomes grown with DM steps. It shows that the tendency of initialised genomes to generate shallow hierarchies comes from the fact that genes starting at the same bit from the duplicated initial sequence tend not to connect, due to the use of the XOR operator (see Section 2). As duplications of the first gene(s) represent the majority of the genes present in the genome, they will not be connected (when choosing an appropriate threshold value), and genes discovered in later DM steps (in smaller numbers) will be highly connected to those earlier genes.

\section{CONCLUSIONS AND FUTURE WORK}

The experimental results obtained with this work show that the use of a gene regulatory network model allows for the construction of network topologies with specific statistical properties, in this case small-world topologies. Once again the initialisation procedure plays a vital role in the seeding of potential models, not only giving them a headstart when compared to random models, but also leading to better final statistical measures, with specific emphasis on clustering coefficient values (as seen in Table 1 ).

This way of evolving topologies also allows one to finetune the objective function, such that specific relationships between $n, k$ and $\ln (n)$ (see Section 3 ) are possible.

A few problems remain with the current approach. The set of weights used in the fitness function (see 4) gives equal importance to the differences between the terms $n, k, \ln (n)$ and 1, regardless of them having a few orders of magnitude of difference. Furthermore, the relationship $C \gg C_{\text {rand }}$, which fundamentally defines a small-world topology, is given the same fitness weight as the differences between the previous four terms. Although there is evolution with this setup, a better fitness function could be designed in the future.

Another interesting future work direction could be a multiobjective approach to the problem. Even more promising could be a multi-objective approach joining the current work and the previous work on scale-free networks [15], evolving topologies which are both scale-free and small-world.

\section{ACKNOWLEDGMENT}

This work was supported by the Sixth European Research Framework (proposal number 034952, GENNETEC project).

\section{REFERENCES}

[1] M. M. Babu, N. M. Luscombe, L. Aravind, M. Gerstein, and S. A. Teichmann. Structure and evolution of transcriptional regulatory networks. Current Opinion in Structural Biology, 14:283-292, 2004.

[2] W. Banzhaf. Artificial regulatory networks and genetic programming. In R. Riolo and B. Worzel, editors, Genetic Programming Theory and Practice, chapter 4, pages 43-62. Kluwer Publishers, 2003.

[3] A.-L. Barabási and R. Albert. Emergence of scaling in random networks. Science, 286(5439):509-512, 1999.

[4] S. N. Dorogovtsev and J. F. F. Mendes. Evolution of Networks: From Biological Nets to the Internet and $W W W$. Oxford University Press, 2003.

[5] M. Garzon. Models of massive parallelism: analysis of cellular automata and neural networks. Springer-Verlag, London, UK, 1995.

[6] M. Giacobini, M. Preuss, and M. Tomassini. Effects of scale-free and small-world topologies on binary coded self-adaptive cea. In J. Gottlieb and G. R. Raidl, editors, Proc. Evolutionary Computation in Combinatorial Optimization, 6th European Conference, EvoCOP, volume 3906 of LNCS, pages 86-98. Springer, 2006.

[7] M. Giacobini, M. Tomassini, and A. Tettamanzi. Takeover time curves in random and small-world structured populations. In H.-G. Beyeretal., editors, Proc. Genetic and Evolutionary Computation GECCO 2005, volume 2, pages 1333-1340. ACM Press, 2005.

[8] J. Guare. Six Degrees of Separation. Vintage, November 1990.

[9] N. Guelzim, S. Bottani, P. Bourgine, and F. Képès. Topological and causal structure of the yeast transcriptional regulatory network. Nature Genetics, 31:60-63, 2002.

[10] H. Jaeger. The echo state approach to analysing and training recurrent neural networks. Technical Report GMD Report 148, German National Research Center for Information Technology, 2001.

[11] H. Jeong, B. Tombor, R. Albert, Z. N. Oltvai, and A.-L. Barabási. The large-scale organization of metabolic networks. Nature, 407:651-654, 2000.

[12] F. Jiang, H. Berry, and M. Schoenauer. Supervised and evolutionary learning of echo state networks. In G. Rudolph et al., editors, Proc. Parallel Problem Solving from Nature - PPSN $X$, volume 866 of LNCS, pages 215-224. Springer-Verlag, 2008.

[13] P. D. Kuo, W. Banzhaf, and A. Leier. Network topology and the evolution of dynamics in an artificial regulatory network model created by whole genome duplication and divergence. Biosystems, 85(3):177-200, 2006.

[14] F. T. Leighton. Introduction to Parallel Algorithms and Architectures: Arrays, Trees, Hypercubes. Morgan Kaufmann, San Mateo, CA, USA, 1992. 


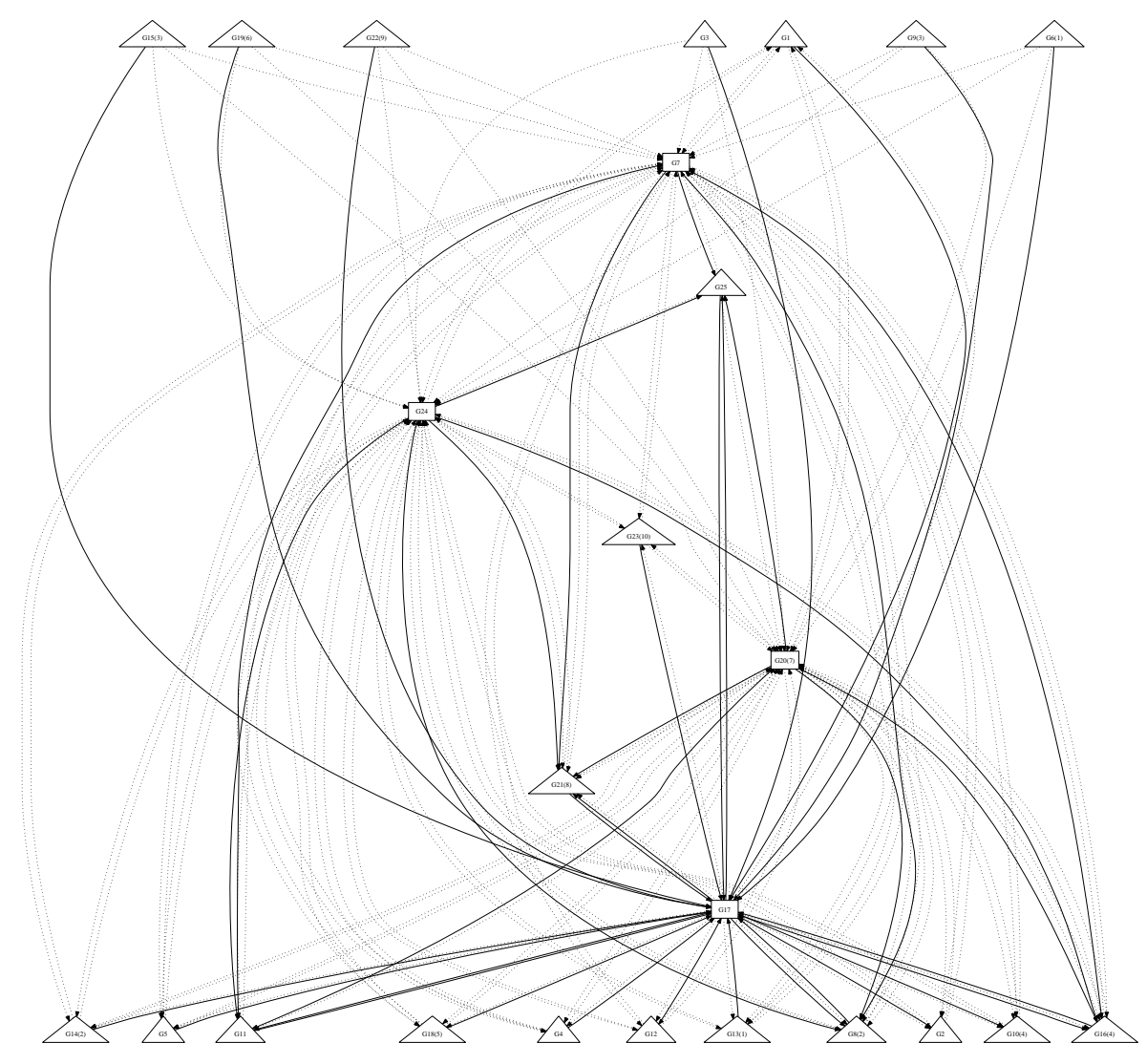

Figure 11: Gene network after 9 duplication events.

Table 2: List of all genes after 10 duplication events.

\begin{tabular}{|c|c|c|c|c|c|c|}
\hline Family & \# genes & $1^{\text {st }}$ loc. & $1^{\text {st }}$ seq. bit & Avg. in & Avg. out & Avg. total \\
\hline Triangle & 39 & 905 & 9 & 9 & 8.8 & 17.8 \\
Quadrangle & 10 & 5713 & 17 & 33 & 31.6 & 64.6 \\
Pentagon & 1 & 27872 & 0 & 37 & 59 & 96 \\
\hline
\end{tabular}

[15] M. Nicolau and M. Schoenauer. Evolving scale-free topologies using a gene regulatory network model. In Proc. IEEE Congress on Evolutionary Computation, CEC 2008, pages 3748-3755. IEEE Press, 2008.

[16] R. Pastor-Satorras, E. Smith, and R. V. Solé. Evolving protein interaction networks through gene duplication. Theoretical Biology, 222:199-210, 2003.

[17] J. L. Payne and M. J. Eppstein. Takeover times on scale-free topologies. In D. Thierens et al., editors, Proc. Genetic and Evolutionary Computation GECCO 2007, pages 308-315. ACM Press, 2007.

[18] J. L. Payne and M. J. Eppstein. The influence of scaling and assortativity on takeover times in scale-free topologies. In M. Keijzer et al., editors, Proc. Genetic and Evolutionary Computation GECCO 2008, pages 241-248. ACM Press, 2008.

[19] I. Rechengerg. Evolutionsstrategie '94. Frommann-Holzboog, Stuttgart, 1994.
[20] V. van Noort, B. Snel, and M. A. Huynen. The yeast coexpression network has a small-world, scale-free architecture and can be explained by a simple model. EMBO Reports, 5(3):280-284, 2004.

[21] D. J. Watts. Small Worlds: The Dynamics of Networks Between Order and Randomness. Princeton University Press, 1999.

[22] D. J. Watts and S. H. Strogatz. Collective dynamics of 'small-world' networks. Nature, 393:440-442, 1998.

[23] K. Wolfe and D. Shields. Molecular evidence for an ancient duplication of the entire yeast genome. Nature, 387:708-713, 1997.

[24] S. Wuchty. Scale-free behavior in protein domain networks. Molecular Biology and Evolution, 18:1694-1702, 2001. 


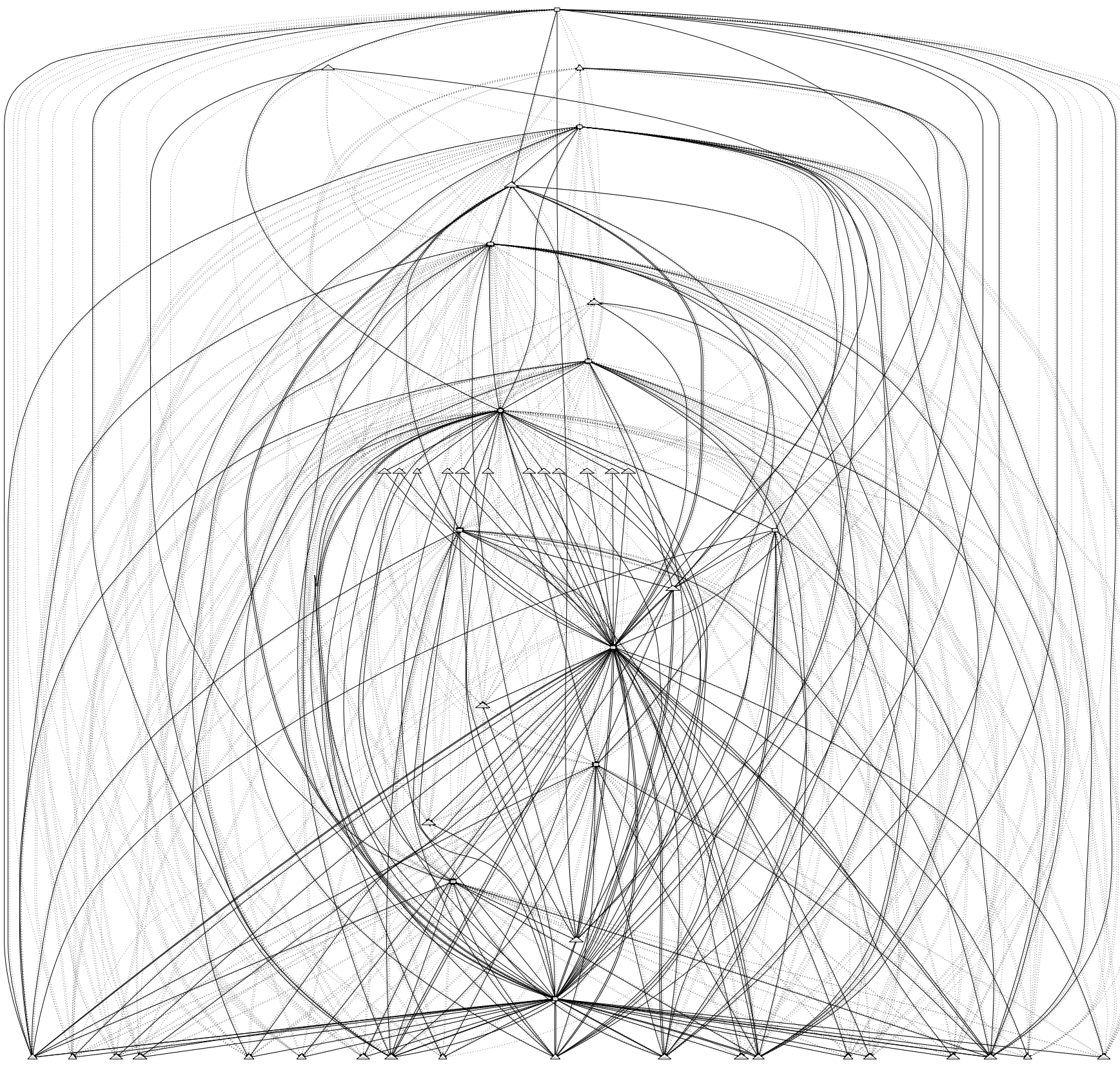

Figure 12: Gene network after 10 duplication events. 